\title{
Gestão da informação científica e tecnológica: relações temáticas dos projetos de iniciação científica da Universidade Federal do Ceará
}

\author{
Gabriela Belmont de Farias \\ Universidade Federal do Ceará, Departamento de Ciências da Informação, Fortaleza, CE, Brasil \\ https://orcid.org/0000-0001-5743-4422 \\ gabibfarias@gmail.com \\ Andreza Pereira Batista \\ Universidade Federal do Ceará, Departamento de Ciências da Informação, Fortaleza, CE, Brasil \\ https://orcid.org/0000-0002-5621-402X \\ andrezapereira028@gmail.com
}

DOI: $\underline{\text { https://doi.org/10.26512/rici.v13.n2.31095 }}$

Recebido/Recibido/Received: 2019-12-19

Aceitado/Aceptado/Accepted: 2020-04-21

Resumo: A produção do conhecimento científico se dá pela interação entre pesquisadores, tendo por objetivo a socialização das informações, com o intuito de auxiliar o desenvolvimento da ciência e da tecnologia visando o desenvolvimento social. No âmbito universitário, a produção do conhecimento científico se dá em diversas modalidades, neste artigo abordaremos as relações estabelecidas pelos projetos do Programa Institucional de Bolsas de Iniciação Científica da Universidade Federal do Ceará. Tais colocações nos impulsionou a desenvolver uma pesquisa visando responder a seguinte questão de partida: Os projetos de pesquisa vinculados ao Programa Institucional de Bolsas de Iniciação Científica da Universidade Federal do Ceará possuem interações entre si permitindo estabelecer relações temáticas entre as comunidades, proporcionando a formação de uma rede de conhecimento científico? Hipótese: Os projetos do Programa Institucional de Bolsas de Iniciação Científica podem gerar conhecimento estratégico que beneficie a produção científica e tecnológica da instituição por meio de suas interações, para atender as demandas no âmbito da pesquisa. Objetivo básico: Identificar a existência de redes de conhecimento científico da Universidade Federal do Ceará, por meio do mapeamento dos projetos visando apresentar as interações temáticas entre as comunidades. Trata-se de uma pesquisa teóricaconceitual e do ponto de vista metodológico a pesquisa é descritiva-exploratória, com abordagem quantiqualitativa, do tipo documental por trabalhar com os projetos científicos. Os resultados permitem inferir que a relação das temáticas abordadas nos projetos científicos, apresentou-se inúmeras possibilidades de dinâmicas de relacionamentos entre as comunidades científicas. Conclui que a análise das características e relações intrínsecas na rede de colaboração poderá servir de subsídios para a comunicação entre os pesquisadores que atuam com temas relacionados, bem como novas possibilidades de relação fortalecendo assim a produção científica e construção do conhecimento visando uma gestão de informação científica que contemple as relações entre as pesquisas e seus benefícios, independente da área de concentração.

Palavras-chave: gestão da informação. informação científica. relações temáticas. organização do conhecimento. 
Scientific and technological information management: thematic relations of scientific initiation projects at the Federal University of Ceará

Abstract: The production of scientific knowledge gives interaction between researchers, aiming at the socialization of information, to assist the development of science and technology and to help social development. At the university level, the production of scientific knowledge takes place in several variations. This article deals with relations with the project rights of the Institutional Program of Scientific Initiation Scholarships of the Universidade Federal do Ceará. These positions encourage us to develop research that addresses the following starting questions: Research projects linked to the Institutional Program of Scientific Initiation Scholarships of the Universidade Federal do Ceará have interactions among themselves and allow social relations to be used among communities, including the formation of a scientific knowledge network? Hypothesis: The projects of the Institutional Program of Scientific Initiation Scholarships can generate strategic knowledge that benefits the institution's scientific and technological production through their interactions, to meet the demands of the research. Basic objective: to identify the presence of scientific knowledge networks of the Universidade Federal do Ceará, through the mapping of projects presented as thematic interactions between communities. This is a theoretical-conceptual and methodological research in a descriptive-exploratory research, with quantitative approach, the documentary type for working with scientific projects. The results indicate that the relationship between approaches in scientific projects shows the various possibilities of relationship dynamics between scientific communities. It is concluded that an analysis of the characteristics and intrinsic relationships in the collaborative network can serve as subsidies for communication between researchers who work with related themes, as well as new possibilities for strengthened relationships, such as scientific production and knowledge construction using a scientific information management that includes the relationships between research and its benefits, regardless of the area of concentration.

Keywords: information management. scientific information. thematic relations. knowledge organization.

Gestión de la información científica y tecnológica: relaciones temáticas de proyectos de iniciación científica en la Universidad Federal de Ceará.

Resumen: La producción de conocimiento científico se produce a través de la interacción entre investigadores, con el objetivo de socializar la información, con el fin de ayudar al desarrollo de la ciencia y la tecnología con el objetivo del desarrollo social. A nivel universitario, la producción de conocimiento científico ocurre en varias modalidades, en este artículo abordaremos las relaciones establecidas por los proyectos del Programa Institucional para Becas de Iniciación Científica en la Universidad Federal de Ceará. Tales ubicaciones nos impulsaron a desarrollar una investigación con el objetivo de responder la siguiente pregunta inicial: Los proyectos de investigación vinculados al Programa Institucional para Becas de Iniciación Científica en la Universidad Federal de Ceará tienen interacciones entre sí, lo que permite el establecimiento de relaciones temáticas entre comunidades, proporcionando la formación de una red de conocimiento cientifico? Hipótesis: los proyectos del Programa de Becas de Iniciación Científica Institucional pueden generar conocimiento estratégico que beneficia la producción científica y tecnológica de la institución a través de sus interacciones, para satisfacer las demandas en el ámbito de la investigación. Objetivo básico: Identificar la existencia de redes de conocimiento científico en la Universidad Federal de Ceará, a través del mapeo de proyectos destinados a presentar interacciones temáticas entre comunidades. Es una investigación teórico-conceptual y, desde un punto de vista metodológico, la investigación es descriptiva-exploratoria, con un enfoque cuantitativo-cualitativo, del tipo documental para trabajar con proyectos científicos. Los resultados permiten inferir que la relación de los temas abordados en los proyectos científicos presentaba numerosas posibilidades de dinámica de las relaciones entre las comunidades científicas. Concluye que el análisis de las características y las relaciones intrínsecas en la red de colaboración puede servir como subsidios para la comunicación entre investigadores que trabajan con temas relacionados, así como nuevas posibilidades de relación, fortaleciendo así la producción científica y la construcción de conocimiento con el objetivo de la gestión de la información científica contempla la relación entre la investigación y sus beneficios, independientemente del área de concentración.

Palabras clave: gestión de la información. información científica relaciones temáticas organización del conocimiento. 


\section{Introdução}

A ciência no Brasil, em seu contexto básico, possui grande parte de sua produção proveniente de pesquisas desenvolvidas em instituições acadêmicas, de modo particular nas universidades públicas.

Segundo dados divulgados pela Web of Science Group acerca da pesquisa no Brasil, o país ocupa a 13a posição no mundo em termos de produção de artigos e revisões de pesquisa indexados na base Web of Science, sendo que as 15 universidades com maior produção de pesquisa, todas públicas, produzem mais de $60 \%$ da produção total de pesquisa (CLARIVATE ANALYTICS, 2019).

Esses dados convergem com o que é visualizado empiricamente pela comunidade científica, em que a pesquisa e consequente produção de comunicações contendo resultados de estudos são focos na disseminação de informações. Segundo Muller (1995), enquanto desenvolve uma pesquisa, o pesquisador precisa ter contato frequente com seus colegas e a literatura de sua área.

Nesse contexto, o contato é realizado por meio de interações com a comunidade científica e suas produções, onde o fazer científico é proveniente de diversas modalidades que incentivam a pesquisa, posto que a produção dá-se por meio das interações entre pesquisadores.

Assim, neste artigo abordaremos uma das modalidades em que a produção do conhecimento científico e as interações entre integrantes dos estudos se fazem presente: os projetos do Programa Institucional de Bolsas de Iniciação Científica (PIBIC). Para tal, temos como questão norteadora da pesquisa: Os projetos de pesquisa vinculados ao PIBIC da Universidade Federal do Ceará (UFC) possuem interações entre si permitindo estabelecer relações temáticas entre as comunidades, proporcionando a formação de uma rede de conhecimento científico?

Temos como hipótese que os projetos do PIBIC podem gerar conhecimento estratégico que beneficie a produção científica e tecnológica da instituição por meio de suas interações, para atender as demandas no âmbito da pesquisa. Assim, temos por objetivo identificar a existência de redes de conhecimento científico da UFC, por meio do mapeamento dos projetos visando apresentar as interações temáticas entre as comunidades existentes na instituição.

Tendo em vista o exposto, para atingir o objetivo proposto, do ponto de vista metodológico, realizamos uma pesquisa descritiva-exploratória, com abordagem quantiqualitativa, do tipo documental por trabalhar com os projetos científicos. Para análise temática, a partir das redes temáticas de interação entre as comunidades científicas (centros, faculdades e instituições). 
Os resultados permitem inferir que a relação das temáticas abordadas nos projetos científicos apresentou inúmeras possibilidades de dinâmicas de relacionamentos entre as comunidades científicas, incluindo-se a construção de redes de relações entre os projetos.

\section{Comunicação científica na universidade}

Nas instituições acadêmicas-científicas a produção de conhecimento científico é tida como função básica. Costa e Pinto (2012) afirmam que desde o início do século XX, as universidades passaram a ser o locus dessa produção no contexto mundial, sendo um local privilegiado de produção e difusão do conhecimento.

Tal produção é realizada, principalmente, mediante o processo de pesquisa científica, em que participam pesquisadores/professores e alunos, encaminhando-se para descobertas, renovação e evolução do conhecimento existente. Para que esse processo de investigação científica seja efetivado, é necessária a divulgação dos resultados obtidos por meio dos estudos, constituindo-se como insumos para novas pesquisas. Nesse contexto, a comunicação da ciência é primordial para seu progresso.

Conforme Targino (2000, p. 10), a “[...] comunicação é um fenômeno natural e intrínseco ao homem, variando de acordo com as características dos grupos nos quais e entre os quais se efetiva", essencial para facilitar os fluxos informacionais, incluindo-se no âmbito da ciência. Os cientistas lançam mão de alternativas para a difusão de seu trabalho, apelando para formas diferenciadas de transmissão, desde recursos mais informais a eletrônicos.

Essencialmente, esses recursos emergem de classificações tradicionais da comunicação científica: formal, estruturada ou planejada; e informal, não estruturada ou não planejada. Ambas consideradas fundamentais à evolução do conhecimento, em seus diversos ambientes e moldes de comunicação (TARGINO, 2000). De acordo com Meadows (1999), a comunicação informal é efêmera, sendo posta a apenas um público limitado, enquanto a formal encontra-se disponível por um longo período de tempo, especialmente em periódicos e livros, tornando-os públicos.

Sob a ótica de Muller (1995, p. 64), a "comunicação é um ato inerente à pesquisa científica", pois no desenvolvimento da ciência, o conhecimento só pode ser aumentado, aprimorado, revisto ou corrigido a partir de resultados de novos estudos. Dessa forma, compreendemos que as comunicações, dentro das instituições de ensino, em particular, as universidades, realizam-se a partir de interações entre os envolvidos no processo: pesquisadores e a comunidade acadêmica ao qual vinculam-se.

Como efeito, compreendemos que a produção científica relaciona-se diretamente com essas comunicações, uma vez que é uma das principais formas de divulgação e disseminação de 
resultados de pesquisas, sendo utilizada como parâmetro para a captação de recursos financeiros para as universidades. Acerca do exposto, Costa e Pinto (2016, p. 19) compreendem que:

\begin{abstract}
A medida de autoridade intelectual de um pesquisador, no mundo acadêmico, é dada pela quantidade de investigações realizadas, pelo número de publicações feitas e pela sua participação em eventos científicos. A publicação e o reconhecimento pelos seus pares, dado pela quantificação da frequência com que o pesquisador é citado torna-se uma verdadeira moeda de valorização.
\end{abstract}

Esse reconhecimento é o que impulsa pesquisadores a comunicarem suas realizações e, dentro do contexto central do artigo, é fundamental para a progressão do conhecimento científico, pois "O mundo científico e acadêmico premia a publicação de resultados de pesquisas, criando para os autores a possibilidade de aumentar o seu reconhecimento social" (Ibid., 2016, p. 20).

A busca pelo reconhecimento social, de acordo com Hagstrom (1979), instiga o desejo dos pesquisadores pela publicação de seus resultados, o que pressupõe trocas constantes de informações entre a comunidade como contrapartida.

Nesse ínterim, os pesquisadores lançam mão de um processo de interações das informações derivadas dos resultados de estudos científicas por meio da comunicação científica, levando ao compartilhamento e impulsionando os fluxos de informação e de conhecimento científico que são decorrentes do movimento em rede na comunidade acadêmico-científica e determinados pelos vínculos que se configuram e reconfiguram.

Essas redes auxiliam no desenvolvimento da ciência e tecnologia (C\&T) para o desenvolvimento social e no ambiente universitário dar-se em diversas modalidades, incluindo no âmbito de programas de bolsas para graduandos, como o Programa Institucional de Bolsas de Iniciação Científica (PIBIC).

\title{
2.1 Programa Institucional de Bolsas de Iniciação Científica (PIBIC)
}

A Iniciação Científica (IC) refere-se a atividade de inserção de estudantes na pesquisa e eventual produção do conhecimento. Massi e Queiroz (2015) afirmam que ela pode ser entendida sob duas perspectivas dentro do ensino superior:

a) Processo que abarca todas as experiências vivenciadas pelo aluno durante a graduação, objetivando promover seu envolvimento com a pesquisa e, por consequência, sua formação científica, e;

b) Desenvolvimento de um projeto de pesquisa elaborado e realizados sob orientação de um docente da universidade, executado com ou sem bolsa para os alunos. 
Ambas as perspectivas complementam-se no contexto universitário, dado que o envolvimento em um projeto de pesquisa acaba por permitir a vivência na pesquisa pelos estudantes de graduação, especialmente em programas voltados para tais fins, como o PIBIC.

O PIBIC, segundo o Conselho Nacional de Desenvolvimento Científico e Tecnológico (CNPq) (BRASIL, 2006), é um programa voltado para o desenvolvimento do pensamento científico e iniciação à pesquisa de estudantes de graduação do ensino superior. Pretende contribuir para a formação de recursos humanos para a pesquisa, além de contribuir para redução do tempo de permanência de alunos na pós-graduação, devido à experiência adquirida nessa modalidade de bolsa, em que:

[...] visa apoiar a política de Iniciação Científica desenvolvida nas Instituições de Ensino e/ou Pesquisa, por meio da concessão de bolsas de Iniciação Científica (IC) a estudantes de graduação integrados na pesquisa científica. A cota de bolsas de (IC) é concedida diretamente às instituições, estas são responsáveis pela seleção dos projetos dos pesquisadores orientadores interessados em participar do Programa. Os estudantes tornam-se bolsistas a partir da indicação dos orientadores. (BRASIL, [20--]).

Desse modo, um projeto PIBIC é desenvolvimento por um professor-orientador em conjunto com um estudante, objetivando realizar uma investigação científica e, possivelmente, produzir publicações científicas dos resultados desses estudos.

Todavia, apontamos que o CNPq possui outros programas de IC aquém os desenvolvidos na universidade, destacando-se os realizados no ensino médio de escolas públicas. A título de informação, exemplificam-se: o Programa de Iniciação Científica Júnior (IC-Jr), o Programa Institucional de Bolsas de Iniciação Científica para o Ensino Médio (PIBIC-EM) e o Programa de Iniciação Científica da Olimpíada Brasileira de Matemática das Escolas Públicas (IC/OBMEP).

Ademais, Costa e Pinto (2016, p. 43) reiteram “[...] que a estratégia do CNPq é a de direcionar a formação do aluno para sua atuação como pesquisador no futuro, além de preparar os estudantes mais promissores para a pesquisa científica", o que compreendemos como indispensável para o desenvolvimento do conhecimento científico.

\section{Metodologia}

O presente artigo é uma pesquisa teórica-conceitual, em que utilizamos para a construção do estudo os pressupostos metodológicos da pesquisa descritiva-exploratória, com abordagem quanti-qualitativa, do tipo documental.

A pesquisa descritiva, de acordo com Gil (2008), tem como principal objetivo a descrição de determinada população ou fenômeno, como é o caso do PIBIC. Já a pesquisa exploratória, ainda segundo o autor, tem por finalidade desenvolver, esclarecer e modificar conceitos e ideias. 
Geralmente, essa pesquisa envolve pesquisa bibliográfica e documental, como é o caso deste artigo.

A pesquisa documental, por sua vez "[...] vale-se de materiais que não receberam ainda um tratamento analítico, ou que ainda podem ser reelaborados de acordo com os objetivos da pesquisa" (GIL, 2008, p. 51). Ela será utilizada por trabalharmos com os projetos científicos PIBIC e seus respectivos relatórios.

O sujeito da pesquisa, como já explanado, é definido pelos projetos aprovados e concluídos do PIBIC da UFC referentes ao período de 2016 / 2017, cujos dados foram cedidos pela Pró-Reitoria de Pesquisa e Pós-Graduação (PRPPG) da instituição, contendo: Centro, Área de Conhecimento, Título, Resumo, Palavras-chave e Produções de cada projeto, com seu quantitativo apresentado no quadro 1.

Quadro 1. Unidades Acadêmicas x Quantidade de Projetos PIBIC 2016 / 2017

\begin{tabular}{|c|c|c|c|c|}
\hline Campus & Unidade Acadêmica & Siglas & $\begin{array}{l}\text { Qt de } \\
\text { Projetos }\end{array}$ & Total \\
\hline \multirow{4}{*}{ BENFICA } & Centro de Humanidades & $\mathrm{CH}$ & 76 & \multirow{4}{*}{135} \\
\hline & Faculdade de Direito & FADIR & 12 & \\
\hline & $\begin{array}{c}\text { Faculdade de Economia, } \\
\text { Administração, Atuária e } \\
\text { Contabilidade }\end{array}$ & FEAAC & 26 & \\
\hline & Faculdade de Educação & FACED & 21 & \\
\hline \multirow{5}{*}{$\mathrm{PICl}$} & Centro de Ciências & $\mathrm{CC}$ & 179 & \multirow{5}{*}{363} \\
\hline & Centro de Clências Agrárias & CCA & 73 & \\
\hline & Centro de Tecnologia & CT & 84 & \\
\hline & Instituto de Cultura e Arte & ICA & 20 & \\
\hline & $\begin{array}{c}\text { Instituto de Educação Fisica } \\
\text { e Esportes }\end{array}$ & IEFES & 07 & \\
\hline \multirow[t]{2}{*}{ PORANGABUSSU } & $\begin{array}{c}\text { Faculdade de Farmácia, } \\
\text { Odontologia e Enfermagem }\end{array}$ & FFOE & 72 & \multirow[t]{2}{*}{156} \\
\hline & Faculdade de Medicina & FAMED & 84 & \\
\hline LABOMAR & Instituto de Ciências do Mar & LABOMAR & 17 & 17 \\
\hline \multirow{4}{*}{ EXTERNO } & Instituto Universidade Virtual & $\begin{array}{l}\text { UFC - } \\
\text { VIRTUAL }\end{array}$ & 05 & \multirow{4}{*}{26} \\
\hline & Campus UFC em Quixadá & $\begin{array}{c}\text { UFC/ } \\
\text { QUIXADÁ }\end{array}$ & 02 & \\
\hline & Campus UFC em Russas & $\begin{array}{l}\text { UFCl } \\
\text { RUSSAS }\end{array}$ & 01 & \\
\hline & Campus UFC em Sobral & $\begin{array}{c}\text { UFCl } \\
\text { SOBRAL }\end{array}$ & 18 & \\
\hline \multicolumn{4}{|c|}{ TOTAL GERAL } & 697 \\
\hline
\end{tabular}

Fonte: Dados cedidos pela Pró-Reitoria de Pesquisa e Pós-Graduação (PRPPG) da UFC (2018)

Na tabela, verificam-se os campis da UFC, em que o Benfica, o Labomar, o Pici e o Porangabuçu são localizados em Fortaleza, capital do estado do Ceará, e o Externo referem-se 
aos campus localizados em outras cidades do estado (Quixadá, Russas e Sobral) e a UFC Virtual. Cada um deles apresenta as suas respectivas unidades acadêmicas com quantidade de projetos.

Por conseguinte, realizamos a leitura dos resumos a partir das comunidades científica, ou seja, por campus, e posterior atribuição de termos controlados partir de tesauros de cada área do conhecimento das unidades acadêmicas demonstradas na tabela 1. Adotamos de três a quatro termos por projeto, com cinco palavras, em média, em que os temas centrais eram buscados nos tesauros e complementados com especificações.

Algumas desses tesauros foram utilizados mais de uma vez ao longo do estabelecimento dos termos, onde usamos quatro tesauros, em média, para cada campus, especificando (ver quadro 2).

Quadro 2. Tesauros utilizados para atribuição dos termos dos projetos PIBIC UFC

\begin{tabular}{|c|c|}
\hline TESAURO & GERENCIAMENTO \\
\hline $\begin{array}{c}\text { Thesaurus Brasileiro da Educação } \\
\text { (Brased) }\end{array}$ & $\begin{array}{c}\text { Instituto Nacional de Estudos e Pesquisas } \\
\text { Educacionais Anísio Teixeira (INEP) - Ministério da } \\
\text { Educação do Brasil }\end{array}$ \\
\hline Biblioteca Nacional ${ }^{1}$ & \begin{tabular}{c} 
Fundação Biblioteca Nacional \\
\hline UNESCO Thesaurus ${ }^{2}$
\end{tabular} \\
\hline $\begin{array}{c}\text { Tesauro Brasileiro de Ciências da } \\
\text { Informação }\end{array}$ & $\begin{array}{c}\text { Organização das Nações Unidas para a Educação, a } \\
\text { Ciência e a Cultura - UNESCO }\end{array}$ \\
\hline $\begin{array}{c}\text { ThesBio - Thesaurus em Biodiversidade Brasileiro de Informação em Ciência e } \\
\text { Tecnologia (IBICT) }\end{array}$ \\
\hline $\begin{array}{c}\text { Tesauro Eletrônico da Biblioteca Virtual } \\
\text { em Saúde }\end{array}$ & $\begin{array}{c}\text { Rede BHL SciELO - Global Biodiversity Heritage } \\
\text { (gBHL) e Scientific Electronic Library Online } \\
\text { (SciELO) }\end{array}$ \\
\hline $\begin{array}{c}\text { Tesauro do Supremo Tribunal Federal } \\
\text { (TSTF) }\end{array}$ & Ministério da Saúde do Brasil \\
\hline Tesauro de Contas Nacional & Supremo Tribunal Federal do Brasil \\
\hline Fonte: Elaborado pelas autoras $(2019)$ & Instituto Rui Barbosa \\
\hline
\end{tabular}

Fonte: Elaborado pelas autoras (2019)

Para mais, elaboramos figuras com as temáticas da comunidade científica integrante dos projetos PIBIC-UFC, contendo os termos mais recorrentes em cada campus da instituição. Todos demonstrados na seção adiante.

\footnotetext{
${ }^{1}$ A Biblioteca Nacional não possui um tesauro propriamente dito, entretanto, apresenta "termos tópicos" que possuem termos gerais, relacionados e específicos, característicos de uma linguagem documentária.

2 O UNESCO Thesaurus não apresenta versão em português, somente em inglês, espanhol, francês e russo. Dessa forma, os termos adotados para cada projeto foram traduzidos e pesquisados em inglês.
} 


\section{Indicativo temático do conhecimento produzido por meio dos projetos PIBIC-UFC}

Os temas dos projeto análisados, do Instututo de Ciências do Mar - (LABOMAR), Instituto Universidade Virtual, Campus Quixada, Russas e Sobral - (EXTERNO) e Centros de Ciência, Ciências Agrárias, Tecnologia, Instituto Cultura e Arte, Educação Fisica e Esporte - (PICI), apresentão relações temáticas nos projetos científicos. Observa-se na figura 1 , uma demanda de estudos relacionados ao desenvolvimento do nordeste, especificamente no ceará no qual as abordagens estão voltadas a sustentabilidade ambiental, agricultira e modelos de negócios sustenteveis - (Labomar e Pici). Verifica-se também que o campus externo dedica a estudos relacionados ao desenvolvimento humano e cultural.

Figura 1 - Temáticas recorrentes Labomar (à esquerda); Externo (centro) e Pici (à direita)
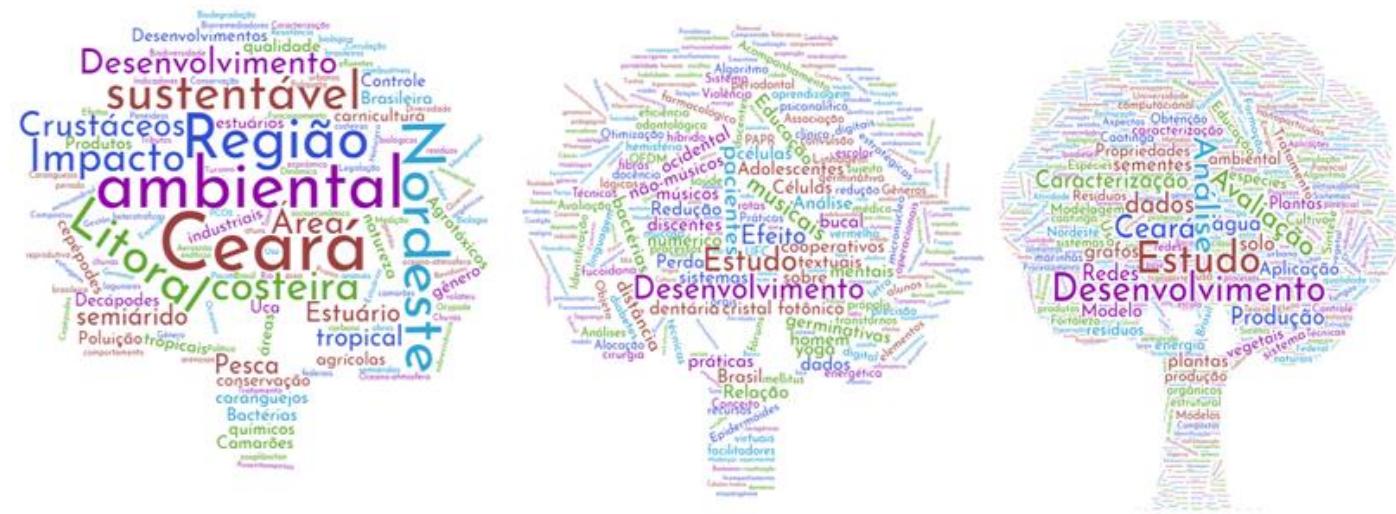

Fonte: Elaborado pelas autoras (2019)

Os temas dos projeto análisados, do Centro de Humanidades, Faculdade de Direito, Economia, Administração, Atuária, Contabilidade, Educação - (Benfica) e a Faculdade de Farmacia, Odontologia, , Enfermagem e Medicina - (Parangabussu) apresentão relações temáticas nos projetos científicos. Observa-se na figura 2, que o Benfica concentra estudos relacionados a ao desenvolvimento humano e social centrado na educação, direito e mediação da informação. As faculdades da área da saúde que estão concentradas no bairro Parangabussu, concentram seus estudos também no desenvolvimento e bem estar humano, no qual verificam práticas relacionadas a redução de mortalidade e doenças como também o desenvolvimento de remédios e tratamentos alternativos dentre outros assuntos. 
Figura 2 - Temáticas recorrentes ao Benfica (à esquerda) e Porangabussu (à direita)
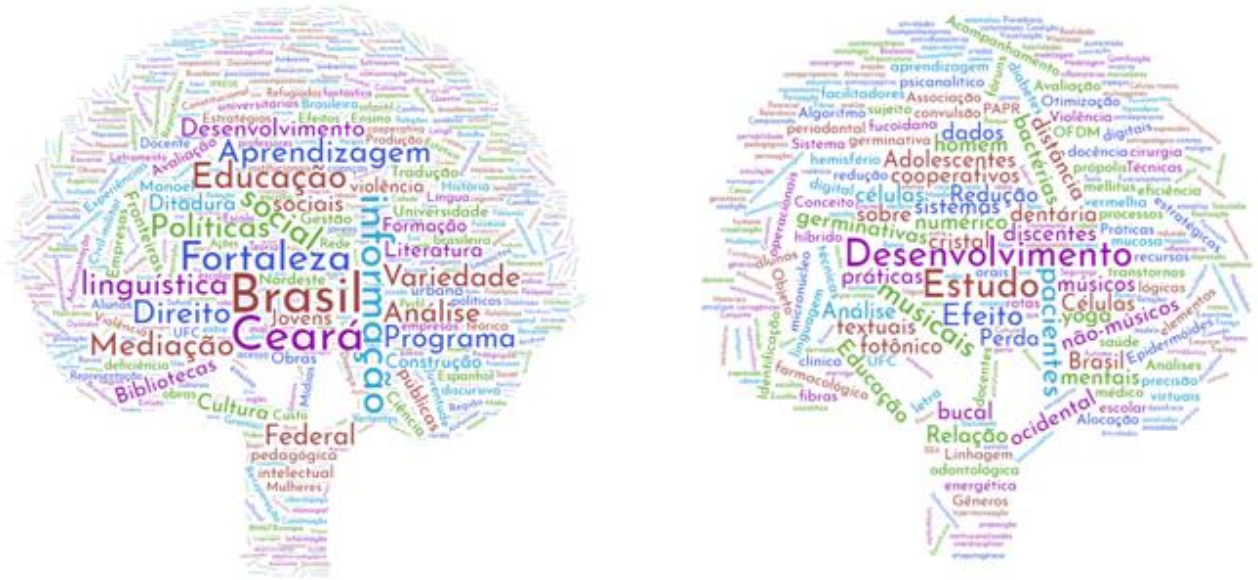

Fonte: Elaborado pelas autoras (2019)

Infere-se que a relação das temáticas abordadas nos projetos científicos, apresentouse inúmeras possibilidades de dinâmicas de relacionamentos entre as comunidades científicas.

\section{Conclusões}

Evidenciou-se que a Biblioteconomia e a Ciência da Informação, por serem áreas do conhecimento que estão relacionadas aos fenômenos informacionais, somadas a aplicação de métodos matemáticos e estatísticos podem contribuir para um gerenciamento mais eficaz da informação científica e tecnológica.

Verificamos que a análise das temáticas caracteza possibilidades de relações intrínsecas possibilitando uma rede de colaboração que poderá servir de subsídios para: a comunicação entre os pesquisadores que atuam com temas relacionados; e a novas possibilidades de relação fortalecendo, assim, a produção científica e construção do conhecimento visando uma gestão de informação científica que contemple as relações entre as pesquisas e seus benefícios, independente da área de concentração.

Por fim, acredita-se que este trabalho contribuiu para uma mapeamento e estabelecimento de redes cientifica na UFC com objetivo de gerar indicadores de análise para identificação da interdisciplinaridade no programa PIBIC-UFC.

\section{Referências}

BRASIL. Conselho Nacional de Desenvolvimento Científico e Tecnológico. Objetivos do programa PIBIC. Brasília, DF: Conselho Nacional de Desenvolvimento Científico e Tecnológico, [20--]. Disponível em: http://www.cnpq.br/pibic Acesso em: 5 nov. 2019.

BRASIL. Conselho Nacional de Desenvolvimento Científico e Tecnológico. Resolução Normativa 
017/2006: bolsas por quota no país. Brasília, DF: Conselho Nacional de Desenvolvimento Científico e Tecnológico, 2007. Disponível em: http://www.cnpq.br/web/guest/view/Ljournal content/56 INSTANCE 0oED/10157/100352 Acesso em: 05 nov. 2019.

COSTA, Airton; PINTO, Adilson Luiz. De bolsista a cientista: a experiência da UFSC com o Programa de Iniciação Científica no processo de formação de pesquisadores (1990 a 2012). Florianópolis: EdUFSC, 2016. 165 p.

CLARIVATE ANALYTICS. Web of Science Group. A pesquisa no Brasil: promovendo a excelência. [S.I.: s. n.], 2019. 42 p. Análise preparada para a CAPES pelo Grupo Web of Science. Disponível em: https://www.sbponline.org.br/arquivos/Promovendo a excele\%CC\%82ncia.pdf Acesso em: 13 set. 2019.

GIL, Antonio Carlos. Métodos e técnicas de pesquisa social. 6. ed. São Paulo: Atlas, 2008.

HAGSTROM, W. O. O controle social dos cientistas. In: DEUS, Jorge Dias de (Org.). A crítica da ciência: sociologia e ideologia da ciência. 2. ed. Rio de Janeiro: Zahar, 1979. p. 81-106.

MARTELETO, Regina Maria. Análise de redes sociais: aplicação dos estudos de transferência da informação. Ciência da Informação, Brasília, v. 30, n. 1, p. 71-81, 2001. Disponível em: http://www.scielo.br/pdf/ci/v30n1/a09v30n1 Acesso em: 17 dez. 2019.

MASSI, Luciana; QUEIROZ, Salete Linhares (Org.). Iniciação científica: aspectos históricos, organizacionais e formativos da atividade no ensino superior brasileiro. São Paulo: Editora Unesp Digital, 2015. Disponível em: http://books.scielo.org/id/s3ny4/pdf/massi9788568334577.pdf Acesso em: 15 nov. 2019.

MEADOWS, Arthur Jack. A comunicação científica. Brasília: Briquet de Lemos, 1999.

MUELLER, Suzana Pinheiro Machado. O crescimento da ciência, o comportamento científico e a comunicação científica: algumas reflexões. Revista da Escola de Biblioteconomia da UFMG, Belo Horizonte, v. 24, n. 1, p. 63-84, 1995. Disponível em: http://www.brapci.inf.br/index.php/article/view/0000002743 Acesso em: 17 dez. 2019.

TARGINO, Maria das Graças. Comunicação científica: uma revisão de seus elementos básicos. Informação \& Sociedade: Estudos, João Pessoa, v. 10, n. 2, p. 37-85, 2000. Disponível em: http://www.brapci.inf.br/index.php/article/view/0000001182/6f78994cb4aa4e65990e1d764d 794d5b Acesso em: 15 nov. 2019. 